\title{
Perfil de pacientes com hérnia de disco em um ambulatório público
}

\author{
The profile of patients with herniated disc in a public clinic
}

Perfil de los pacientes con hernia discal en un ambulatorio público

Gabriel Gomes da Silva1, Francisca Cecilia Viana Rocha ${ }^{2,1}$, Maria Zélia de Araújo Madeira ${ }^{3}$, Ivonizete Pires Ribeiro ${ }^{4,1 *}$.

\section{RESUMO}

Objetivo: Descrever o perfil dos pacientes com hérnia de disco em um ambulatório público da capital piauense. Métodos: Trata-se de estudo descritivo com abordagem quantitativa, conduzido por 345 individuos de ambos os sexos, atendidos nos setores de neurologia e ortopedia com confirmação do diagnóstico de hérnia de disco. Utilizou-se para coleta de dados um questionário. Estes foram analisados e processados no programa SPSS Statistics 20. Resultados: Foi evidenciado que a maioria é do sexo feminino $64,06 \%$, com idade de 41 a 60 anos $(56,81 \%)$, baixo nível de escolaridade $(67,54 \%)$, pardos $(49,28 \%)$, renda familiar até um salário mínimo $(93,91 \%)$, hipertensos $(51,59)$; sedentários $(50,72 \%)$ e sobrepeso $(48,97 \%)$. Conclusão: Conclui-se que a hérnia de disco está relacionado a fatores de riscos como más posturas ergonômicas, alimentação inadequada ou associada a algumas comorbidades existentes. Porém a doença pode ser controlada por meio de posturas corretas e controle de doenças que contribuem para o desenvolvimento ou agravamento da hernia de disco. Nesse sentido, a melhor forma de prevenção consiste em mudança do estilo de vida, orientação a ser dada na atenção básica.

Palavras-chaves: Enfermagem, Hérnia de disco, Pérfil de saúde, Ambulatório, Fatores de risco.

\begin{abstract}
Objectives: To describe the profile of patients with herniated disc in a public clinic in the city of Piauí. Methods: It is a descriptive research with quantitative approach led by 345 people from both gender, assisted in the neurology and orthopedics sectors with confirmation of herniated disc diagnosis. A questionnaire was carried out as a way to collect data. Those data were analyzed and processed in the SPSS Statistics 20 program. Results: It was possible evidence that the majority of patients observed is female $64,06 \%$, ages from 41 to 60 years old $(56,81 \%)$ low level of schooling $(67,54 \%)$ brown colour $(49,28 \%)$ family income up to a minimum wage $(93,91 \%)$, they are hypertensive $(51.59)$; sedentary $(50,72 \%)$ and overweight (48,97\%). Conclusion: It was concluded that herniated disc is related to risk factors such as poor ergonomic postures, inadequate diet or associated with some existing comorbidities. Although the disease can be controlled through correct postures and control of diseases that contribute to the development or worsening of the herniated disc. Therefore the best form of prevention consists of lifestyle changing, which is an orientation to be given in primary care.
\end{abstract}

Keywords: Herniated disc, Health Profile, Public Clinic, Risk Factors.

\section{RESUMEN}

Objetivo: Describir el perfil de los pacientes con hernia discal en un ambulatorio publico de la capital piauiense. Métodos: Se trata de un estudio descriptivo con abordaje cuantitativa, conducido por 345 individuos de ambos los sexos, atendidos en los sectores de neurología y ortopedia con confirmación del

\footnotetext{
${ }^{1}$ Graduando de Enfermagem do Centro Universitário Uninovafapi. Teresina-Piauí.

${ }^{2}$ Mestre em Enfermagem pela UFPI. Teresina - Piauí.

${ }^{3}$ Ciências Médicas/UNICAMP, Educação/UFPI, Docente da graduação em enfermagem, Docente da Pós-graduação em Ciência e Saúde/UFPI.

${ }^{4}$ Universidade Federal de Goiás/UFG. Teresina - Piauí. *E-mail: Ivonizeteribeiro@gmail.com
}

SUBMETIDO EM: 6/2019

ACEITO EM: 7/2019

PUBLICADO EM: 7/2019 
diagnostico de hernia discal. Fue utilizado para la recolección de datos un cuestionario. Estos fueron analizados y procesados en el programa SPSS Statistics 20. Resultados: Fue evidenciado que la mayoría es del sexo femenino, $64,06 \%$, con edad de 41 a 60 años $(56,81 \%)$, bajo nivel de escolaridad $(67,54 \%)$, pardos $(49,28 \%)$, renta familiar hasta un sueldo mínimo $(93,91 \%)$, hipertensos $(51,59 \%)$, sedentarios $(50,72 \%)$ y sobrepeso $(48,97 \%)$. Conclusión: Se concluye que la hernia discal está relacionada a factores de riesgos como mala postura ergonómica, alimentación inadecuada o asociada a algunas comorbilidades existentes. Pero la enfermedad puede ser controlada por medio de posturas correctas y el control de enfermedades que contribuye para el agravamiento de la hernia discal. En este sentido la mejor manera de prevención consiste en cambio del estilo de vida, orientación a ser dada en la atención básica.

Palabras claves: Enfermería, Hernia discal, Perfil de salud, Ambulatorio, Factores de riesgo.

\section{INTRODUÇÃO}

A hérnia de disco é uma patologia causada nos discos que compõe a coluna vertebral sendo caracterizada como uma desordem musculoesquelética responsável por manifestar algias, em virtude da ruptura do anel fibroso e consequente deslocamento do núcleo pulposo, comprimindo assim as terminações e raízes nervosas, mas pode vir na forma assintomática. É uma doença crônica degenerativa considerada como uma síndrome multifatorial, com grandes impactos econômicos e emocionais e de alta prevalência, chegando a afastar os indivíduos ativos de suas atividades sociais e laborais (PAZZINATO MF, et al., 2014).

Os profissionais de enfermagem estão diretamente ligados ao cuidado com o paciente e acabam esquecendo-se de cuidar de si próprio. Comportamentos e posturas inadequadas durante a assistência ao paciente requerem esforços físico e repetitivos, estes ocasionam prejuízo na saúde desses profissionais ao longo da vida, isto leva-os a ausências freqüentes no trabalho comprometendo assim o cuidado continuado (ALENCAR MCB, 2012).

A patologia constitui um problema de saúde pública e as estimativas da literatura revelam que pode ser controlada tendo em vista que fatores como o processo de envelhecimento, o sedentarismo, má postura no ambiente de trabalho, movimentos repetitivos, o excesso de peso também prejudica as estruturas da coluna vertebral e a rotina das atividades de vida diária afetam no desenvolvimento da hérnia de disco e/ou em seu agravamento (COSTA MC, et al., 2015).

Segundo Furtado RNV, et al. (2014). a dor lombar afeta $80 \%$ da população ao ponto de onerar os sistemas de saúde e da previdência em termos de diagnósticos, tratamento, absenteísmo e aposentadoria prematura. As dores na coluna vertebral geralmente acometem a região lombar em algum momento na vida das pessoas e isso se dá devido hábitos posturais inadequado no trabalho, em suas atividades diárias ou em qualquer local que esteja e que tenha uma postura incorreta, pois essas dores são a principal causa de incapacidade em indivíduos com menos de 45 anos de idade, desse modo, apresentando alto índice de incapacidade e morbidade em indivíduos economicamente ativos.

Os fatores de risco no desenvolvimento da hérnia de disco estão relacionados a fatores individuais e profissional. O fator individual se refere a idade, sexo, índice de massa corporal, desequilíbrio muscular, capacidade de força muscular, condições socioeconômicas, degeneração dos discos devido ao excesso de e esforço físico e presença de outras patologias. Já os fatores de riscos profissionais dizem respeito a posturas inadequadas durante a rotina de trabalho, posturas adotadas na realização de tarefas, além das condições de funcionamento dos equipamentos disponíveis, sem falar nas formas de organização execução do trabalho (FORTES CGMNB, et al., 2013).

Vale ressaltar que a incapacidade laborativa de seus portadores compromete a qualidade de vida, dificuldade de realizar suas atividades diárias do dia a dia. Portanto a melhor forma de evitar que a mesma cause prejuízo no cotidiano e atividades desenvolvidas é a prevenção, com adoção de hábitos saudáveis como praticar exercícios físicos, ter uma alimentação adequada, evitar fazer atividades que demandem grandes esforços físico, evitar movimentos repetitivos (ALMEIDA ICGB, et al., 2008). O presente estudo objetivou identificar e analisar o perfil sociodemograficos dos pacientes com hérnia de disco. 


\section{MÉTODOS}

Trata-se de uma pesquisa de caráter descritivo, com abordagem quantitativa realizada em um ambulatório de caráter público, junto aos pacientes atendidos e acompanhados nas especialidades de neurologia e ortopedia situado na cidade de Teresina/PI, Brasil. O ambulatório é referência no Estado do Piauí como centro de excelência, para o tratamento de hérnia de disco. Foram incluídos no estudo, pacientes que deram entrada no ambulatório público nos setores ortopédicos e neurológicos com confirmação de hérnia de disco e que se disponibilizarem em participar da coleta de dados realizadas no período de agosto a outubro de 2018 e excluídos os pacientes com suspeita de hérnia de disco sem comprovação diagnostica. Para a coleta dos dados utilizou-se um questionário que contemplou as seguintes variáveis: idade, sexo, peso, IMC, estado civil, hábitos de vida (tabagismo, etilismo, sedentarismo), nível de escolaridade, renda familiar, hereditariedade. Além das variáveis foi contemplado as comorbidades, tipo de trabalho, fatores psicológicos e psicossociais. Os dados coletados foram tabulados inicialmente numa planilha Microsoft Excel e então exportada para o programa IBM SPSS Statistics 20 para o processamento e o teste estatístico e analisados de forma descritiva univariavel em frequências absolutas (№) e relativas (\%). A pesquisa respeitou os princípios éticos preconizados pela Resolução 466/12 da Comissão Nacional de Ética em Pesquisa com o número CAAE 2.698.053

\section{RESULTADOS}

A maioria era do sexo feminino $64,06 \%$, com faixa etária predominante entre 41 e 60 anos $56,81 \%$, e em quase sua totalidade procedentes do Piauí, com nivel de escolaridade ensino fundamental incompleto, casados, pardos e renda familiar baixa (Tabela 1).

Tabela 1 - Perfil sóciodemográfico dos pacientes. Teresina (PI), Agosto - Outubro 2018.

\begin{tabular}{|c|c|c|c|}
\hline & & № $=345$ & $\%$ \\
\hline \multirow{4}{*}{ Sexo } & Mas & 124 & 35,94 \\
\hline & Fem & 221 & 64,06 \\
\hline & $<18$ & 1 & 0,29 \\
\hline & 18 a 23 & 1 & 0,29 \\
\hline \multirow{5}{*}{ Faixa etária (anos) } & 24 a 30 & 10 & 2,90 \\
\hline & 31 a 40 & 14 & 4,06 \\
\hline & 41 a 60 & 196 & 56,81 \\
\hline & Mais de 60 & 123 & 35,65 \\
\hline & PI & 324 & 93,91 \\
\hline \multirow[t]{4}{*}{ Procedência } & MA & 11 & 3,19 \\
\hline & CE & 10 & 2,90 \\
\hline & Ens. fund. incomp. & 233 & 67,54 \\
\hline & Ens. fund. comp & 50 & 14,49 \\
\hline \multirow{6}{*}{ Escolaridade } & Ens.médio incomp. & 11 & 3,19 \\
\hline & Ens.médio comp. & 40 & 11,59 \\
\hline & Ens. sup. incomp. & 3 & 0,87 \\
\hline & Superior & 8 & 2,32 \\
\hline & Solteiro & 57 & 16,52 \\
\hline & Casado & 174 & 50,43 \\
\hline \multirow[t]{5}{*}{ Estado civil } & União estável & 66 & 19,13 \\
\hline & Divorciado & 17 & 4,93 \\
\hline & Viúvo & 31 & 8,99 \\
\hline & Branca & 95 & 27,54 \\
\hline & Preta & 79 & 22,90 \\
\hline \multirow{4}{*}{ Cor / raça } & Parda & 170 & 49,28 \\
\hline & Amarela & 0 & 0 \\
\hline & Indígena & 1 & 0,29 \\
\hline & Ate 1 & 324 & 93,91 \\
\hline \multirow{2}{*}{ Renda Familiar } & 1 e 3 & 21 & 6,09 \\
\hline & Acima de 3 & 0 & 0 \\
\hline
\end{tabular}

Fonte: Pesquisa direta, 2018. 
As comorbidades encontradas nos indivíduos entrevistados mostrou-se que mais da metade eram diabéticos 51,6\%, a segunda comorbidade existente nos pacientes eram osteoporose com 29\% (Tabela 2).

Tabela 2 - Presença de comorbidades nos pacientes. Teresina (PI), Agosto-Outubro 2018.

\begin{tabular}{llll}
\hline & & $\mathbf{N}=(\mathbf{3 4 5})$ & $\%$ \\
\hline Osteoporose & Sim & 100 & 28,99 \\
Diabetes & Não & 245 & 71,01 \\
& Sim & 52 & 15,07 \\
& Não & 293 & 84,93 \\
Artrose & Sim & 178 & 51,59 \\
& Não & 167 & 48,41 \\
& Sim & 48 & 13,91 \\
& Não & 297 & 86,09 \\
& Bico de papagaio & 1 & 0,29 \\
& Glaucoma & 1 & 0,29 \\
& Depressão & 1 & 0,29 \\
& Bursite & 2 & 0,58 \\
& Mal Parkinson & 2 & 0,58 \\
& Hepatite & 1 & 0,29 \\
& Varizes & 1 & 0,29 \\
Outras doenças & Aneurisma & 1 & 0,29 \\
& Asma & 2 & 0,58 \\
& Gastrite & 2 & 0,58 \\
& Colesterol & 4 & 1,16 \\
& Artrite & 9 & 2,61 \\
& Enxaqueca & 1 & 0,29 \\
& Escoliose & 1 & 0,29 \\
& Reumatismo & 2 & 0,58 \\
& Semoutrasdoenças & 314 & 91,01 \\
\hline
\end{tabular}

Fonte: Pesquisa direta, 2018.

Tabela 3 - Hábitos de vida dos pacientes associados a hérnia de disco. Teresina (PI), Ago-Out 2018.

\begin{tabular}{llll}
\hline & & $\mathbf{N}=\mathbf{3 4 5}$ & $\%$ \\
\hline Atividade física & Sim & 127 & 36,81 \\
& Não & 218 & 63,19 \\
& Caminhada & 95 & 27,54 \\
Tipo de atividade física & Academia & 27 & 7,83 \\
& Hidroginástica & 3 & 0,87 \\
& Dança & 2 & 0,58 \\
Sedentarismo & Nãopratica & 218 & 63,19 \\
& Sim & 175 & 50,72 \\
Tabagista & Não & 170 & 49,28 \\
& Sim & 63 & 18,26 \\
Etilista & Não & 282 & 81,74 \\
Atividade física com esforço & Sim & 85 & 24,64 \\
& Não & 260 & 75,36 \\
& Sim & 54 & 15,65 \\
& Não & 291 & 84,35 \\
Tipo de esforço físico & Capinar & 7 & 2,03 \\
& Pegar peso & 32 & 9,28 \\
& Acordeom & 1 & 0,29 \\
& Costurar & 1 & 0,29 \\
& Plantar & 1 & 0,29 \\
& Quebrar coco & 1 & 0,29 \\
& Faxinar & 3 & 0,87 \\
& Roça & 6 & 1,74 \\
& Pegar boi & 2 & 0,58 \\
& Não faz esforço & 291 & 84,35 \\
\hline
\end{tabular}

Fonte: Pesquisa direta, 2018. 
Sobre os hábitos de vida associado a hernia de disco, evidenciou-se que grande maioria eram sedentários com $50,7 \%$ e o tipo de atividade que era mais praticado era a caminhada com apenas 27, 5\% (Tabela 3).

Tabela 4 - Conhecimento da doença, hospitalização, realização de fisioterapia, cirurgia e uso de medicamentos. Teresina (PI), Agosto - Outubro 2018

\begin{tabular}{|c|c|c|c|c|c|c|}
\hline & & №= $=345$ & $\%$ & Média & Mínimo & Máximo \\
\hline \multirow{2}{*}{ Conhecimento da doença } & Sim & 251 & 72,75 & & & \\
\hline & Não & 94 & 27,25 & & & \\
\hline \multirow{2}{*}{ Hospitalização } & Sim & 137 & 39,71 & & & \\
\hline & Não & 208 & 60,29 & & & \\
\hline \multicolumn{2}{|l|}{ № de hospitalizações } & & & 3 & 1 & 15 \\
\hline \multirow{2}{*}{ Realiza fisioterapia } & Sim & 181 & 52,46 & & & \\
\hline & Não & 164 & 47,54 & & & \\
\hline \multirow{2}{*}{ Realizou cirurgia } & Sim & 8 & 2,32 & & & \\
\hline & Não & 337 & 97,68 & & & \\
\hline \multirow{10}{*}{ Uso de medicação } & Sim & 142 & 41,16 & & & \\
\hline & Não & 203 & 58,84 & & & \\
\hline & Voltarem & 7 & 2,03 & & & \\
\hline & Prednisona & 2 & 0,58 & & & \\
\hline & Tandrilax & 5 & 1,45 & & & \\
\hline & Ibuprofeno & 6 & 1,74 & & & \\
\hline & Dipirona & 9 & 2,61 & & & \\
\hline & Tramal & 18 & 5,22 & & & \\
\hline & Beta trinta & 27 & 7,83 & & & \\
\hline & Mioflex-A & 3 & 0,87 & & & \\
\hline \multirow{10}{*}{ Tipo de medicação } & Prolopa & 1 & 0,29 & & & \\
\hline & Nimesulida & 17 & 4,93 & & & \\
\hline & Torsilax & 5 & 1,45 & & & \\
\hline & Tandene & 8 & 2,32 & & & \\
\hline & Duoflam & 1 & 0,29 & & & \\
\hline & Acoxia & 8 & 2,32 & & & \\
\hline & Dorflex & 17 & 4,93 & & & \\
\hline & Diclofenaco & 5 & 1,45 & & & \\
\hline & Meloxicam & 2 & 0,58 & & & \\
\hline & Não usa & 203 & 58,84 & & & \\
\hline
\end{tabular}

Fonte: Pesquisa direta, 2018.

Grafico 1- Estado nutricional dos pacientes com hérnia de disco

\section{Estado nutricional dos pacientes}

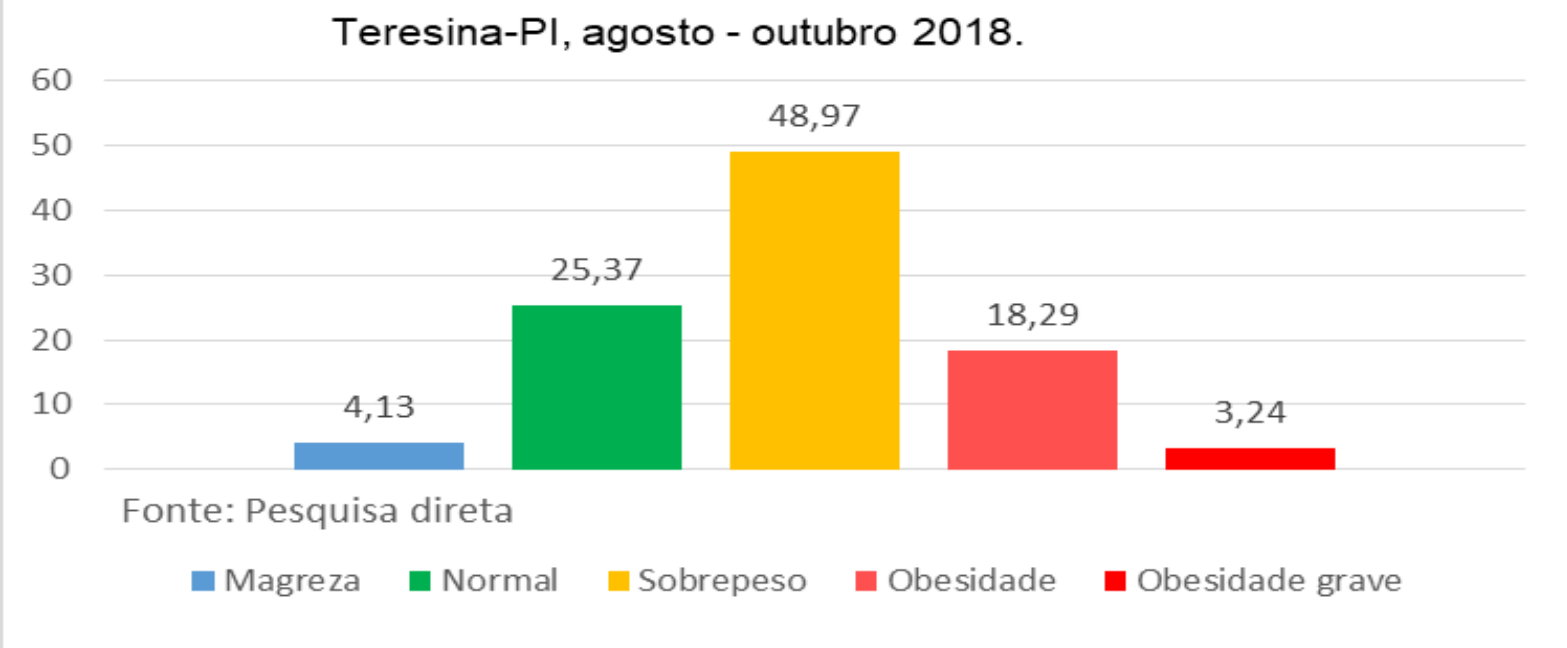

Fonte: Pesquisa direto, 2018.

REAS | Vol. Sup.29 | e1071 | DOI: https://doi.org/10.25248/reas.e1071.2019 Página 5 de 8 
De acordo com gráfico, a maioria dos entrevistados tiveram sobrepeso 48,9\%. O estado nutricional se deu por meio da classificação do Índice de Massa Corporal (IMC) (Gráfico 1). Levando em consideração que esse dado se dar pela falta de exercicios praticadas pelos os individuos, além de ser um fator de risco no desenvolvimento de doenças que acometem a coluna ou consequências as pessoa que já sofrem com algum problema nessa região.

\section{DISCUSSÃO}

O estudo mostrou que a hérnia de disco é um problema de saúde que afeta diretamente a vida das pessoas, está relacionado ao estilo de vida, levando-os a vários graus de incapacidade e prejuízos em seu cotidiano, comprometendo assim a sua qualidade de vida.

Prevaleceu no estudo o sexo feminino, isto se deve pelo fato da mulher ter menor massa muscular e óssea, menor resistência muscular ou até mesmo articulações mais frágeis, menos adaptada a elevadas esforços físicos (LINS VML, et al.,2013).

Bottamedi X, et al. (2017), em seu estudo evidenciou a incidência de hérnia de disco em lavradores em um determinado município na zona rural do Piauí, a média de idade é de 46 a 55 anos. Nessa pesquisa a idade média foi de 41 a 60 anos, caracterizando assim um perfil mais jovem do encontrado na literatura, isso mostra que, com o avanço da idade é comum o aumento dos processos degenerativos resultando em desgastes das estruturas articulares.

A baixa escolaridade evidenciada no estudo interfere no acesso às informações para correção de hábitos de vida saudáveis, posturas adequadas em seus ambientes de trabalho. no aprendizado em relação a sua saúde (COSTA MC, et al.,2015).

De acordo com a pesquisa de Furtado RNV, et al. (2014) o estado civil tem prevalência elevada em indivíduos casados, concordando com outros estudos. A postura incorreta no trabalho ou até mesmo em domicilio constitui um fator de risco. Nos indivíduos entrevistados foram detectados índice elevado de pessoas pardas, entretanto este fato, pode se dar por conta da região geográfica e suas características climáticas, que podem provocar modificações da população em relação a essa variável. Outra variável de extrema importância é a renda familiar que teve o salário mínimo com grande prevalência nos participantes da pesquisa. Porém não foi encontrado nenhum autor que discuta a relação dessas duas variáveis citadas em relação com a doença.

A presença de comorbidades nos pacientes com hérnia de disco, dentre as doenças com maior prevalência está a hipertensão arterial sistêmica $51,6 \%$ e osteoporose com $28 \%$ e em menor quantidade a diabetes $15 \%$. Estudo como o de Ghisleni MM, et al., (2014), que pesquisaram o perfil epidemiológico dos pacientes atendidos na área de ortopedia e traumatologia da clínica-escola de fisioterapia também foi observada, grande prevalência de hipertensão, diabetes ou da presença de ambas, e acompanhado da hérnia de disco. Estas associações podem ser explicadas pelos fatores de risco que tais doenças possuem em comum, entre eles o envelhecimento e estilo de vida adotada em grande maioria favorecem 0 desenvolvimento das mesmas.

Para Bandeira F e Carvalho EF (2007), que fala sobre a prevalência de osteoporose e fraturas vertebras em mulheres na pós menopausa identificaram alta índice de osteoporose em região lombar nessas mulheres acometida por essa doença com faixa etária 50 anos. Esse mesmo estudo revela que o exercício físico exerce um importante efeito na saúde óssea dos indivíduos, principalmente daqueles que se aproximam de sua idade idosa, e quando associado a ingesta adequada de cálcio melhora ainda mais o fortalecimento ósseo e evita fraturas óssea.

Visto que a osteoporose é uma doença que afeta especialmente o sexo feminino, é importante enfatizar que isso se deve em virtude da perda acentuada de massa óssea nas mulheres devido ao período da perimenopausa que acontece devido a diminuição de um dos principais hormônios feminino o estrogênio (SANTOS GCP, et al., 2017). 
Foi evidenciado que cerca de $63 \%$ dos participantes não praticam exercícios físicos a qual ocasiona a perda de massa muscular e óssea. Para Bottamedi X, et al., (2016), o sedentarismo é indicador de risco, portanto a prática de atividade física, melhora o reforço muscular, a flexibilidade e o condicionamento aeróbico, podendo oferecer efeitos positivos à autonomia funcional dos indivíduos, especialmente, entre os com mais de 60 anos.

Para Vialle LR, et al., (2010), foi verificado alto índice de fumantes entre a população com dor lombar. Porém, a prevalência de tabagista nesse estudo foi baixa, pelo fato destes já terem abandonado o habito de fumar. Apesar do baixo número de indivíduos que realizavam atividades que demandem grande força física na pesquisa, estudos encontrados na literatura comprovam que essas atividades é um dos fatores de risco, por apresentar desgastes articulares ao longo dos anos, podendo levar indivíduos a terem a ruptura do núcleo pulposo que se localizam nos discos que compõe a coluna vertebral a qual irá comprimir as terminações nervosas presentes (ALBUQUERQUE AB, et al., 2013).

Mais de $72 \%$ dos participantes da pesquisa comprovam ter conhecimento acerca da patologia, ponto positivo para a prevenção dos fatores desencadeantes no desenvolvimento dessa doença. Para Fortes CGMNB, et al., (2013), A média do número de sessões de fisioterapia é de 22. Outros estudos apontaram médias de 14 a 20 sessões fisioterapêuticas. Outras pesquisas avaliaram que as expectativas do paciente frente ao tratamento fisioterapêutico, confirmam o alívio da dor (FERREIRA MS e NAVEGA MT, 2010). Isso mostra que o número de fisioterapia realizada pelos indivíduos da pesquisa se mostra inferior comparada a outras pesquisas, desse modo, torna-se evidente uma quantidade menor de sessões realizada e um resultado mais rápido alcançado.

$58 \%$ relataram não fazer uso de medicação, e os que utilizam fazem uso de anti-inflamatórios, analgésicos e corticoides, por serem medicamentos de custo acessível e de fácil acesso aos usuários. Isso mostra que o tratamento cirúrgico é uma última escolha e acontece estágio avançado da doença. Souza FFR, et al., (2011), em sua pesquisa mostrou que a infiltração com corticosteroides associada a anestésicos locais possui um efeito agudo no tratamento, com melhora significativa.

Almeidal ICGB et al., (2008), afirma que a obesidade é outro fator associado a dor lombar e outros problemas que afetam a coluna. O sobrepeso pode provocar o desequilíbrio biomecânico do corpo, alterando o eixo de gravidade e, consequentemente, aumentando o recrutamento da musculatura antigravitacional, ocasionando a dores lombares. Cerca de 48\% tiveram IMC acima do que se classifica como normal, resultado que corrobora com outros estudos sobre a mesma temática.

Vale ressaltar, que existem lacunas de estudos sobre a temática, a qual observa-se que há necessidade de desenvolver novas pesquisas que facilite elaborar estratégias preventivas, de modo que os profissionais tenham conhecimento e possam conscientizar e promover saúde a população. A pesquisa teve limitações, como a restrição de ter sido realizada apenas em um ambulatório, deixando assim de abranger realidades de outros serviços e de diferentes outras regiões do país. Essas considerações são de extrema relevância, pois a ampliação de campos e participantes dará mais incentivo para que a temática abordada sejam aprofundadas e possibilite discussões com propósito de sugerir estratégias de intervenções que gerem reflexos na qualidade da assistência de Enfermagem a esses pacientes.

\section{CONCLUSÃO}

A hérnia de disco, é uma patologia que tem um bom prognostico, seu tratamento é basicamente clinico, com uso de analgésicos, anti-inflamatórios e fisioterapia. É uma doença que pode ser prevenida com mudança no estilo de vida, modificação do hábito alimentar, pratica de exercícios físicos, correção da postura no trabalho e domicilio, bem como evitar excesso de peso. Os resultados apontam que a mulher é mais favorável, estando presente também o sedentarismo, obesidade, doenças crônicas não transmissível e o baixo nível de escolaridade. A pesquisa contribuiu, pois os resultados evidenciaram que por meio da educação em saúde e informações adequadas, é possível evitar a doença. Essa promoção da saúde começa na atenção primária por ser a primeira porta de entrada dos pacientes. Portanto a equipe de saúde deve estar preparada para fazer as orientações devidas para a prevenção. 


\section{AGRADECIMENTOS}

Agradeço a instituição Hospital Getúlio Vargas (HGV) por ter autorizado a coleta dos dados no ambulatório do hospital.

\section{REFERÊNCIAS}

1. ALBUQUERQUE AB, et al. Manifestações clínicas da hérnia discal cervical. Revista Acta Méd, 2013: 34(6): 7-17.

2. ALMEIDA ICGB, et al. Prevalência de dor lombar crônica na população da cidade de Salvador. Rev Bras Ortop, 2008; 43(3): 96-102.

3. ALENCAR MCB, TERADA TM. O afastamento do trabalho por afecções lombares: repercussões no cotidiano de vida dos sujeitos. Revista de Terapia Ocupacional da Universidade de São Paulo, 2012; 23(1): 44-51.

4. BANDEIRA F, CARVALHO EF. Prevalência de osteoporose e fraturasvertebrais em mulheres na pós-menopausa atendidas em serviços de referência. Revista Brasileira de Epidemiologia, 2007; 10(1): 86-98.

5. BOTTAMEDI $X$, et al. Programa de tratamento para dor lombar crônica baseado nos princípios da EstabilizaçãoSegmentar e na Escola de Coluna. Rev. Bras. Med. Trab, 2016; 14(3): 206-213.

6. BRASIL. Resolução oํ 466/2012, sobre pesquisa envolvendosereshumanos. Ministério da Saúde, 2012.

7. COSTA MC, et al. Perfil epidemiológico e clínico dos pacientes com queixa de dor lombar atendidos em uma clínica escola de fisioterapia. Revista Movimenta, 2015; 12(15): 37-42.

8. FERREIRA MS, NAVEGA MT. Efeitos de um programa de orientação para adultos com lombalgia. Acta Ortopédica Brasileira, 2010; 15(4): 127-131.

9. FORTES CGMNB, et al. Manifestações clínicas da hérnia discal lombar. Acta méd, 2013; 34(7): 2-7.

10. FURTADO RNV, et al. Dor lombar inespecífica em adultos jovens: fatores de risco associados. Revista Brasileira de Reumatologia, 2014; 54(5): 371-377.

11. GHISLENI MM, et al. Perfil epidemiológico dos pacientes atendidos naárea de ortopedia e traumatologia da clínicaescola de fisioterapia Univates. Revista DestaquesAcadêmicos, 2014; 6(3): 117-125.

12. LINS VML, et al. Avaliação da qualidade de vida nos portadores de hérnia de disco lombar praticantes do métodopilates. Terapia Manual, 2013; 11(54): 567-572.

13. PAZZINATTO MT. Identificação dos métodos cirúrgicos utilizados para correção de hérnia discal lombar no Hospital Universitário do Oeste do Paraná. Rev Bras Pesq Saúde, 2014; 16(4): 131-135.

14. SANTOS GCP, et al. A osteoporose e seu acometimento em idosos e sua relação com as quedas. Revista Saúde em Foco, 2017; 6(5): 364-369.

15. SOUZA FFR. Estudo clínico da eficácia do bloqueio anestésico radicular transforaminal no tratamento da radiculopatia lombar. Coluna/Columna, 2011; 10(3): 234-238.

16. VIALLE LR, et al. Hérnia discal lombar. Rev Bras Ortop, 2010; 1(45): 17-22. 